\title{
Mechanical and Morphological Properties of Lignin-Based Thermosets
}

Marcus E. Jawerth, Calvin J. Brett, Cédric Terrier, Per T. Larsson, Martin Lawoko, Stephan V. Roth, Stefan Lundmark, and Mats Johansson*

Cite This: ACS Appl. Polym. Mater. 2020, 2, 668-676

Read Online

ACCESS | Llll Metrics \& More | 回 Article Recommendations ｜ＳＳupporting Information

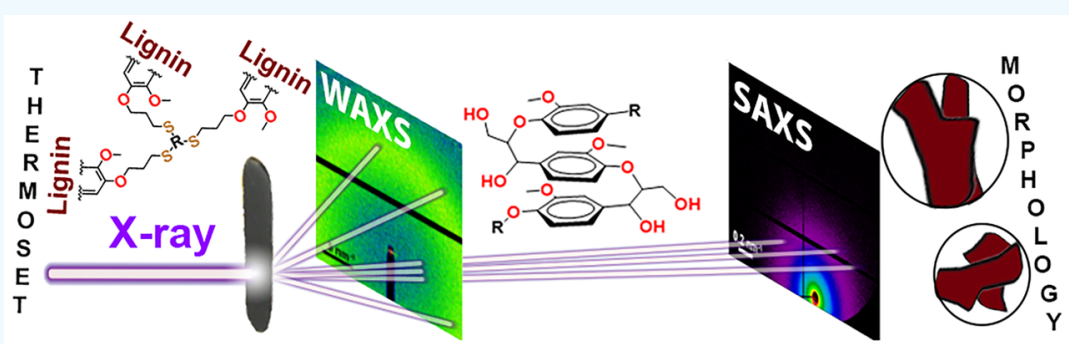

ABSTRACT: The need for renewable alternatives for fossil-based aromatic material constituents is evident for a more sustainable society. Lignin is the largest source of naturally occurring aromatic compounds but has mainly been considered as waste material or energy source in the pulp and paper industry. Developments in extracting lignin from these processes provide a large source for renewable aromatic structures to be used in various applications. Producing thermosets out of lignin is a very promising route to utilize this raw material toward, for example, composite application. The buildup of the molecular network based on oligomeric lignin segments will be different from traditional thermoset analogues, where the constituents often are smaller molecules, and will have an effect on the material properties. In this work LignoBoost Kraft lignin is refined, chemically modified, and used to produce freestanding thermosets with different architectures and properties. These different thermosets are evaluated, and the possibilities to tailor the material properties through work-up and modification are demonstrated. Morphological studies on the formed thermosets using X-ray scattering show systematic differences in molecular stacking and aggregate sizes.

KEYWORDS: lignin fractions, aryl allyl ethers, thiol-ene thermoset, mechanical properties, small- and wide-angle X-ray scattering

\section{INTRODUCTION}

Lignin is an abundant, aromatic, naturally occurring biopolymer that has gained, and is gaining, increased interest for material applications. ${ }^{1-8}$ This is because there is a drive to find suitable, renewable replacements for crude-oil-based aromatic monomers such as bisphenol A. ${ }^{2,3}$ The aromatic structure of lignin provides a rigid component to polymeric systems forming high- $T_{\mathrm{g}}$ materials, and its phenolic as well as aliphatic hydroxyl functionalities could be used as chemical handles in various applications. ${ }^{2,9,10}$ The molecular rigidity providing stiffness is crucial in applications such as, for example, composites where the modulus is a key property. ${ }^{11}$ High performance composite thermoset matrices furthermore represent a suitable application where the color of the material is of less importance. Typical thermosets used as matrices for fiber reinforced composites have a modulus ranging from 0.7 $\mathrm{GPa}$ (polyurethane) to $5.0 \mathrm{GPa}$ (bismaleimides) and $\mathrm{T}_{\mathrm{g}}$ 's ranging from $65^{\circ} \mathrm{C}$ (epoxy) to $370{ }^{\circ} \mathrm{C}$ (polyimide). ${ }^{11}$ Utilizing lignin as a raw material is not, however, a trivial task. Lignin is inherently heterogeneous in its native form where three main monolignols can be linked in various different ways and will vary in ratio depending on plant species. ${ }^{12}$ Furthermore, the processes applied to extract lignin from biomass alter the structure of the lignin backbone. For example, in the Kraft process, the most commonly used process when producing pulp, lignin is separated from its biomass by using harsh physical and chemical conditions. ${ }^{2,13}$ This causes the lignin backbone to be cleaved, fragmented, and recombined, making it almost nonrecognizable from its source in nature. ${ }^{13-16}$ Furthermore, new functional groups are introduced through, for example, oxidation reactions resulting in the formation of carboxylic acids, aldehydes, and ketones. ${ }^{2,14}$ Nevertheless, technical lignin is produced in a megaton scale ${ }^{2}$ and provides a steady stream of raw material for new potential products. The LignoBoost process is one route designed to retrieve lignin from the black liquor of the Kraft process by precipitation. ${ }^{17}$

To overcome the challenges regarding the heterogeneity different approaches can be used to achieve more easy-to-

Received: October 22, 2019

Accepted: January 21, 2020

Published: January 21, 2020 
handle fractions in subsequent steps, including solvent fractionation $^{18,19}$ and ultrafiltration. ${ }^{20,21}$ One example of such a fractionation procedure to fractionate Kraft lignin with respect to size was developed in our laboratory by Duval et al. in $2015^{18}$ using solvents that are considered green and benign. $^{22}$ Fractionating the lignin by solubility is practical when working in lab scale and straightforwardly introduces the possibility to work under homogeneous reaction and mixing conditions.

Incorporating lignin in material systems has been examined in various ways ranging from filler components ${ }^{23-25}$ over thermoplastics $^{26}$ to thermosets. ${ }^{27-30}$ This often requires chemical modification of the lignin to introduce chemical handles for further reactions or to compatibilize it with, for example, polymer matrices. The multifunctionality of lignin makes it versatile in terms of possibilities for chemical modification. In previous work an allylation procedure, adding allyl ether functionality selectively on the phenols of ethanol soluble LignoBoost Kraft lignin, was developed, and the product was used as a constituent in thermosetting resins by using thermally induced thiol-ene chemistry. ${ }^{27,31}$

Conventional thermosets are normally based on thermosetting resins made from combinations of uniform monomers. This means that aromatic units are normally single aromatic monomers that are relatively evenly distributed throughout the thermoset structure, for example, in two-component epoxy/ amine resins. ${ }^{32}$ Starting from lignin fractions instead will make the distribution of these aromatic segments more heterogeneous. How this will affect the final thermoset properties is not fully known, but it may introduce nanoscale heterogeneities into the thermoset. Heterogeneities, such as density fluctuations, can also be formed in thermoset polymers, especially in chainwise polymerizing systems, and have an effect on the material properties. ${ }^{33,34}$ Stepwise polymerizing systems such as the thiol-ene cross-linking used in the present work are less prone to this. ${ }^{37,38}$ It is thus of interest to reveal whether the use of lignin fractions introduces these structure fluctuations and how these affect any structure-property relationship. Here, Xray scattering techniques come into play, allowing to quantify the morphology of the material and relate it to its macroscopic properties, for example, mechanical properties. ${ }^{36,37}$

In this work the selective allylation procedure was performed on four different LignoBoost Kraft lignin fractions obtained by using solvent fractionation. ${ }^{18}$ The modified fractions were cured by using the thermally induced thiol-ene cross-linking reaction previously described. ${ }^{27}$ The present study has been focused on the understanding of the significance of the choice of individual lignin fractions for the properties of thermoset materials. The formed thermosets have been evaluated in terms mechanical properties and morphological aspects to determine how work-up of lignin can be used to tailor material properties.

\section{EXPERIMENTAL SECTION}

Materials. LignoBoost Kraft lignin (KL) has been provided by Stora Enso. Ethyl acetate (EtOAc), ethanol (EtOH), methanol $(\mathrm{MeOH})$, and acetone was aquired through VWR Chemicals. Sodium carbonate $\left(\mathrm{Na}_{2} \mathrm{CO}_{3}\right)$, allyl chloride (98\%), and trimethylolpropane tris(3-mercaptopropionate) (3MP3 TMP, 95\%) were purchased from Sigma-Aldrich. Silicone molds were prepared from a Sylgard elastomer and curing agent (10:1).

Methods. Nuclear Magnetic Resonance (NMR). ${ }^{1} \mathrm{H}$ and ${ }^{31} \mathrm{P}$ NMR were recorded at room temperature on a Bruker Avance III HD $400 \mathrm{MHz}$ instrument with a BBFO probe equipped with a Z-gradient coil for structural analysis. Data were processed with MestreNova (Mestrelab Research) by using $90^{\circ}$ shifted square sine-bell apodization window; the baseline and phase correction was applied in both directions.

${ }^{31} \mathrm{P}$ NMR samples were prepared and analyzed based on the procedure reported by Argyropolous et al. in 1994. ${ }^{39}$ A more thorough description is found in the Supporting Information.

Size Exclusion Chromatography (SEC). THF SEC analyses were performed using a GPCMAX and autosampler from Malvern Instruments equipped with RI detector and ran at $35{ }^{\circ} \mathrm{C}$ with a flow rate of $1.0 \mathrm{~mL} \mathrm{~min}{ }^{-1}$. The columns used was a guard column, TGuard, and two linear mixed bead columns, LT4000L, and a conventional calibration with polystyrene standards and a flow rate of

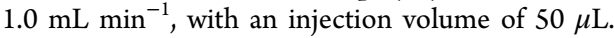

Fourier Transform Infrared Spectroscopy (FT-IR). The measurements were performed using a PerkinElmer Spectrum 100 FT-IR with a triglycine sulfate (TGS) detector equipped with a MKII Golden Gate single reflection ATR system of Specac Ltd. All spectra were recorded in the range of $600-4000 \mathrm{~cm}^{-1}$ with 16 scans averaged at $4.0 \mathrm{~cm}^{-1}$ resolution at room temperature. All data were analyzed by using PerkinElmer Spectrum software V10.5.1.

Density Measurements of Thermosets. The densities of the thermosets were measured by using a density determination kit from Mettler Toledo (Precisa XR2055M-DR) for AX/AT/AG and PG-S/ PG/PR balances.

Dynamical Mechanical Analysis (DMA). Mechanical analysis was performed on a TA Instruments DMA Q800 in tensile mode. The geometry of the sample was roughly $12 \times 6 \times 0.5 \mathrm{~mm}^{3}$. The starting temperature was set to $-50{ }^{\circ} \mathrm{C}$, where it was held isothermally for 10 min before heating with a rate of $3{ }^{\circ} \mathrm{C} \min ^{-1}$ up to $210{ }^{\circ} \mathrm{C}$. The frequency was set to $1 \mathrm{~Hz}$ with a strain amplitude of $95 \%$ of $0.1 \%$ of the sample length. The measurements were performed on quadruplicates or triplicates of each sample depending on amount of available resin.

Thermogravimetric Analysis (TGA). The analysis was performed using a TA Instruments Hi-Res TGA 2950 analyzer under nitrogen flow at a heating rate of $10{ }^{\circ} \mathrm{C} \mathrm{min}^{-1}$ between 40 and $700{ }^{\circ} \mathrm{C}$. The sample size was between 5 and $10 \mathrm{mg}$.

Scattering Techniques. Small- and wide-angle X-ray scattering (SAXS/WAXS) experiments were performed to study the morphological inner structure of the thermosets. The SAXS experiments were performed at beamline P03 at the storage ring PETRA III (Hamburg, Germany).$^{40}$ The used wavelength was $\lambda=0.987 \AA$, and the sampleto-detector distance $(S D D)=4889 \pm 1 \mathrm{~mm}$. The $2 \mathrm{D}$ scattering patterns were recorded by using a photon counting pixel detector Pilatus $1 \mathrm{M}$ (Dectris AG, Switzerland) (pixel size $=172 \mu \mathrm{m}^{2}$ ) and analyzed with DPDAK v. $1.2 .^{41}$

SAXS and WAXS X-ray measurements were performed on an Anton Paar SAXSpoint 2.0 system (Anton Paar, Graz, Austria) equipped with a Microsource $\mathrm{X}$-ray source $(\mathrm{Cu} \mathrm{K} \alpha$ radiation, wavelength $\lambda=1.5418 \AA$ ) and a Dectris 2D CMOS Eiger R $1 \mathrm{M}$ detector with $75 \times 75 \mu \mathrm{m}^{2}$ pixel size. All measurements were performed with a beam size of $\sim 500 \mu \mathrm{m}$ diameter, at a sample stage temperature of $25{ }^{\circ} \mathrm{C}$ with a beam path pressure at 1-2 mbar. For WAXS the SDD was $110.8 \mathrm{~mm}$, and for SAXS the SDD was 575.7 $\mathrm{mm}$. All samples were mounted on a sampler for solids on a heated sampler and a VarioStage (Anton Paar, Graz, Austria). The samples were exposed to vacuum during measurement. For each sample five frames each of $4 \mathrm{~min}$ duration were read from the detector, giving a total measurement time of $20 \mathrm{~min}$ per sample. For all samples the transmittance was determined and used for scaling of the scattering intensities. The software used for instrument control was SAXS drive version 2.01.224 (Anton Paar, Graz, Austria), and postacquisition data processing was performed by using the software SAXS analysis version 3.00.042 (Anton Paar, Graz, Austria).

Procedures. Fractionation. The LignoBoost Kraft lignin (KL) was washed with deionized water at $60^{\circ} \mathrm{C}$ for $1 \mathrm{~h}$. The dispersion was filtered to separate the washing media from the KL, and the washing step was repeated until the $\mathrm{pH}$ of the aqueous permeate had increased 


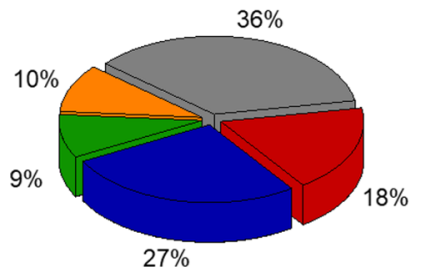

\begin{tabular}{|c|c|c|c|}
\hline $\mathrm{KL}_{\text {EtOAC }}$ & $M_{n} \approx 300$ & $M_{w} \approx 1000$ & Đ 3.1 \\
\hline $\mathrm{KL}_{\text {EtOH }}$ & $M_{n} \approx 800$ & $M_{w} \approx 2700$ & Đ 3.3 \\
\hline $\mathrm{KL}_{\mathrm{MeOH}}$ & $M_{n} \approx 900$ & $M_{w} \approx 3700$ & Đ 4.0 \\
\hline $\mathrm{KL}_{\text {Acetone }}$ & $M_{n} \approx 1500$ & $\mathrm{M}_{\mathrm{w}} \approx 7600$ & Đ 5.1 \\
\hline ble & Not & IF & \\
\hline
\end{tabular}

Figure 1. One typical relative mass distribution of washed LignoBoost Kraft lignin $(\mathrm{KL})$ when following the solvent fractionation procedure and the molecular weights and distributions as determined by SEC using THF as solvent.

to about 5.5 , after which the $\mathrm{KL}$ were dried in a vacuum oven at $80^{\circ} \mathrm{C}$ overnight.

Roughly $25 \mathrm{~g}$ of washed lignin was dispersed in $300 \mathrm{~mL}$ of EtOAc in a beaker equipped with magnetic stirring. The suspension was stirred at room temperature for $2 \mathrm{~h}$ before the lignin fraction that had not dissolved was recovered from the mixture by filtration. The nonsoluble residue was washed with additional EtOAc and then left to dry in the fume hood. The EtOAc soluble fraction was retrieved by removing the solvent under reduced pressure at $40{ }^{\circ} \mathrm{C}$. The sticky residue was resolubilized in acetone before being precipitated in deionized water to obtain a water dispersion. The dispersion was finally freeze-dried, and a fine brown powder was obtained. The procedure was repeated with the EtOAc insoluble lignin fraction by using first $\mathrm{EtOH}$, then $\mathrm{MeOH}$, and lastly acetone to achieve five different lignin fractions, namely, one ethyl acetate-soluble fraction $\left(\mathrm{KL}_{\mathrm{EtOAc}}\right)$, one ethanol-soluble fraction $\left(\mathrm{KL}_{\mathrm{EtOH}}\right)$, one methanolsoluble fraction $\left(\mathrm{KL}_{\mathrm{MeOH}}\right)$, one acetone-soluble fraction $\left(\mathrm{KL}_{\text {Acetone }}\right)$, and one insoluble fraction $\left(\mathrm{KL}_{\text {Insoluble }}\right)$ with the next fraction exhibiting a larger molecular weight than the previous one as determined by SEC.

Selective Allylation. The allylation procedure was based on the procedure described in our earlier work ${ }^{27,31}$ with a few adjustments. $\mathrm{Na}_{2} \mathrm{CO}_{3}$ is used instead of $\mathrm{NaOH}$ for milder reaction conditions and thus a more fail-safe procedure. The allylation was performed on the different fractions obtained from the previous step excluding the "insoluble" fraction. The "insoluble" fraction was excluded since green and relatively volatile solvents where used in the mixing of resins and solvent casting steps. The molar ratio between the reagents were 1:1.4:1.4 with regards to lignin phenols: $\mathrm{Na}_{2} \mathrm{CO}_{3}$ :allyl chloride, and the solvent used was a $\mathrm{EtOH}(96 \%) /$ water mixture (60/40). A general procedure is described as follows:

The $\mathrm{Na}_{2} \mathrm{CO}_{3}$ was predissolved in deionized water before being mixed with the EtOH to form the reaction media. The corresponding $\mathrm{KL}_{\text {Fraction }}$ (fraction referring to the solvent used to extract it, EtOAc, $\mathrm{EtOH}, \mathrm{MeOH}$, or acetone) was added to the alkaline solution under agitation and was dissolved while heating to the reaction temperature at $65{ }^{\circ} \mathrm{C}$. When the lignin appeared dissolved and the reaction temperature had been reached, the allyl chloride was added through a septum with a syringe. The mixture was left to react for $\sim 40 \mathrm{~h}$ before additional deionized water was added to the mixture to dilute the system. $\mathrm{HCl}(0.1 \mathrm{M})$ was added dropwise to lower the $\mathrm{pH}$ of the solution and precipitate the product which was recovered by filtration. The product was washed two times with deionized water to remove traces of acid and salts. The product was then redissolved in acetone and reprecipitated in deionized water to obtain a water dispersion before being freeze-dried, and the allylated product $\left(\mathrm{A}-\mathrm{KL}_{\text {Fraction }}\right)$ could be recovered as a light brown powder. The product was last dried in a vacuum oven at $80{ }^{\circ} \mathrm{C}$ overnight to dry the product.

The allylation reaction was confirmed to be efficient for all fractions by ${ }^{31} \mathrm{P}$ NMR, ${ }^{1} \mathrm{H}$ NMR, and FT-IR. The conversions of the reactions were calculated to be around $90 \%$ for all different fractions except for $\mathrm{KL}_{\text {Acetone, }}$ which had a conversion of around $80 \%$, through ${ }^{31} \mathrm{P}$ NMR signal integrals. The yields were estimated to be in the range of 75$90 \%$ where most of the losses occurs during the work-up of the product in the filtration and freeze-drying steps.

Thermosetting of Thiol-Ene Resin. Resins were prepared similarly to what has been reported previously ${ }^{27}$ by using an $\approx 60 \mathrm{wt} \%$ ratio of allylated lignin to achieve samples with similar lignin content.
To be able to perform mechanical analysis, freestanding pieces had to be prepared. The allylated lignin fractions were mixed with $3 \mathrm{MP} 3$ TMP as a cross-linker using EtOAc as a cosolvent. For the two fractions $\mathrm{A}-\mathrm{KL}_{\mathrm{MeOH}}$ and $\mathrm{A}-\mathrm{KL}_{\text {Acetone }} 10-20$ vol $\%$ of EtOH was added as a cosolvent to fully dissolve the components.

The resins were used to fill a soft silicon mold from which the thermosets could be removed without breaking. Because of the high viscosity of the lignin/cross-linker mixture the cosolvent (EtOAc/ $\mathrm{EtOH}$ ) had to be evaporated slowly to avoid bubble formation inside the bulk material, especially for the two latter fractions. To achieve this, the drying was performed in the fume hood covered by a glass Petri dish.

When the cosolvent had evaporated and homogeneous resin samples had been obtained ( $\left.\mathrm{R}-\mathrm{KL}_{\text {Fraction }}\right)$, the mold was placed in an oven and heated to $125{ }^{\circ} \mathrm{C}$ and left to cure for $20 \mathrm{~h}$. The cured samples, now forming a thermoset $\left(\mathrm{T}-\mathrm{KL}_{\text {Fraction }}\right)$, where then allowed to cool to room temperature before carefully being removed from the mold. The samples were smoothened by using sandpaper to even out the sample shapes for the mechanical analysis which demands as uniform cross section as possible. The curing of the resins was confirmed by FT-IR, the mechanical properties were examined using DMA, and TGA was used to study the thermal stability.

Thermoset Density Calculations. The densities where calculated by weighing the cured thermoset samples in air as well as in deionized water at 22 and $23.5^{\circ} \mathrm{C}$ by using eq 1 according to Archimedes' principle.

$$
\frac{m_{\text {in air }}}{m_{\text {in air }}-m_{\text {in deionized water }}}\left(\rho_{\text {deionized water }}-\rho_{\text {air }}\right)+\rho_{\text {air }}
$$

Scattering Measurements of the Thermosets. SAXS and WAXS techniques were used to examine the morphology of the cured thermoset materials. During the SAXS measurements at P03 beamline, the beam illuminated the thermoset under normal incidence, and the transmission signal was recorded. All samples were scanned laterally to avoid beam damage and checked for homogeneity. To extract the morphology quantitatively, the data were subsequently plotted in $\ln I(q) / q^{2}$ and fitted in the linear regime to gain the Guinier radius, $R_{\mathrm{g}}$ (often termed the radius of gyration in scattering measurements $\left.{ }^{42}\right)$ from within the thermoset. Hence, the slope is a direct measure for the $R_{\mathrm{g}}$; slope $=R_{\mathrm{g}}^{2} / 3$.

The WAXS patterns achieved by the lab X-ray source at RISE were radially integrated, and the occurring signal was fitted by using two distinct Gaussian functions. The center of each signal was further used to calculate the observed distance $d=2 \pi / q$, were $q$ is the scattering vector. The integrals of the signals where used to calculate the ratio between the populations within the sample.

\section{RESULTS AND DISCUSSION}

Fractionation and Modification. The adopted solvent fractionation procedure of LignoBoost Kraft lignin (KL) yields five different fractions, four of which are soluble in organic solvents that are considered green and benign, the fifth fraction being the insoluble residue. ${ }^{22}$ The sequential solvent fractionation is designed to produce fractions with different properties and increasing molecular mass with each step in the 

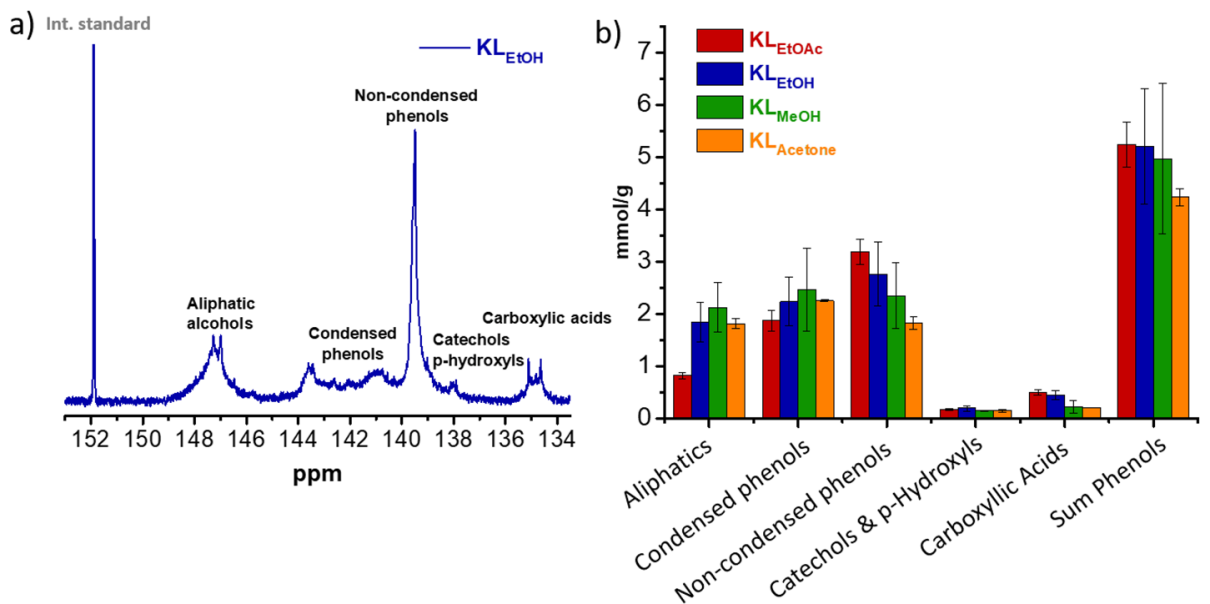

Figure 2. ${ }^{31} \mathrm{P}$ NMR spectrum with assignments (a) and the functional group abundance based on signal intensity integration (b).

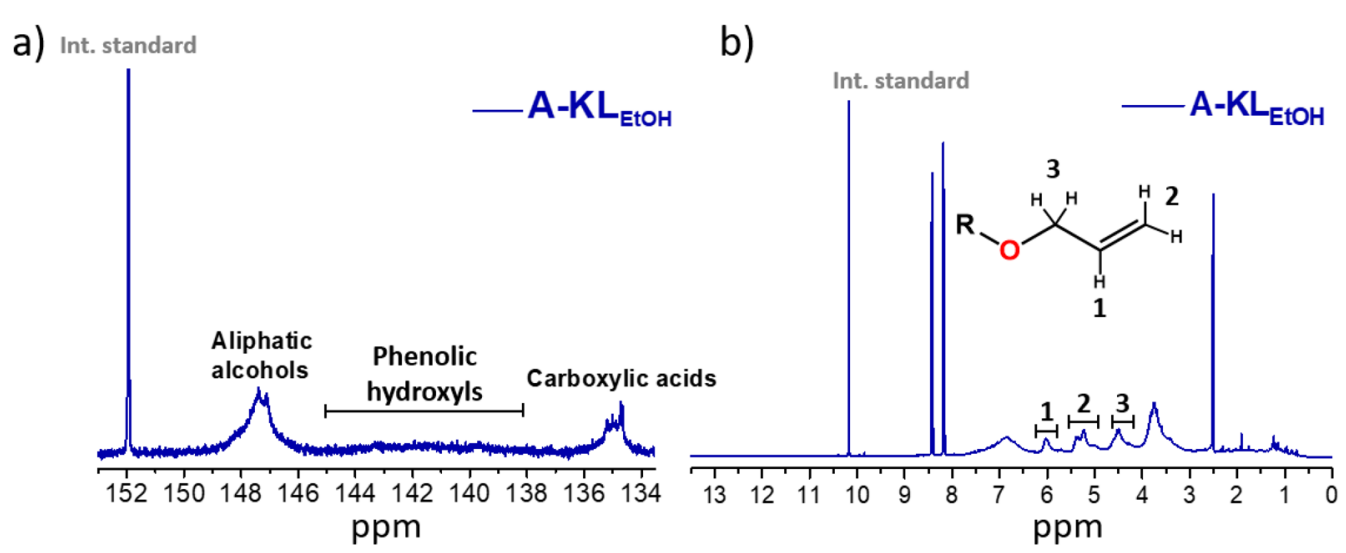

Figure 3. ${ }^{31} \mathrm{P}$ NMR (a) and ${ }^{1} \mathrm{H}$ NMR (b) spectra of the allylated product (A- $\mathrm{KL}_{\mathrm{EtOH}}$ ). The signals corresponding to phenolic hydroxyls in the $\mathrm{P}$ NMR spectra (143.5 to $136 \mathrm{ppm}$ ) have disappeared, and the newly formed signals in the H NMR (between 4 and 6 ppm) show the formation of the aryl allyl ether.

process. ${ }^{18}$ A typical relative fraction mass distribution and SEC data for this work is presented in Figure 1.

The fractionation procedure is optimized to render fractions with increasing molecular weight for each step of the process; ${ }^{18}$ however, the fractions differ from each other in more aspects than that. Through ${ }^{31} \mathrm{P}$ NMR it was possible to measure the abundance of different hydroxyl groups present in the lignin fraction. Although one should be aware of the limited precision of this analytical method, ${ }^{43}$ it is a very useful tool when exploring the abundance of different functionalities in a lignin sample. A representative ${ }^{31} \mathrm{P}$ NMR spectrum $\left(\mathrm{KL}_{\mathrm{EtOH}}\right)$ and a summary of functional group abundance in the different fractions are represented in Figure 2.

After the different fractions had been produced and analyzed, a selective allylation reaction was performed. The procedure was efficient for the fractions due to their full solubility in the reaction media (alkali EtOH/water mix). The allylation procedure was evaluated using ${ }^{1} \mathrm{H}$ and ${ }^{31} \mathrm{P}$ NMR (Figure 3) as well as FT-IR. It can clearly be seen that the signals corresponding to the phenolic hydroxyl have all disappeared in the ${ }^{31} \mathrm{P}$ NMR spectra after the allylation and at the same time new signals appeared in ${ }^{1} \mathrm{H}$ NMR spectra. The signals between 6.3 and $4 \mathrm{ppm}$ in the ${ }^{1} \mathrm{H}$ NMR spectra correspond to the added protons of the allyl group. ${ }^{31}$ NMR spectra for all samples are found in the Supporting Information (Figures S1 and S2).
The degree of allylation was calculated by the conversions of phenols obtained by ${ }^{31} \mathrm{P}$ NMR and through quantification of allyl groups by ${ }^{1} \mathrm{H}$ NMR for comparison. Although the techniques differ in that ${ }^{31} \mathrm{P}$ NMR analysis requires a phosphorylation reaction of the samples and ${ }^{1} \mathrm{H}$ NMR have a risk of small overlaps, similar results were obtained. The results are shown in Table S1.

Curing of the Thermosets. The solubility of the lignin is affected by the functionalization due to the phenol capping. This is the reason why pure EtOAc and EtOAc with $\sim 10$ vol \% of $\mathrm{EtOH}$ were sufficient as solvents when mixing the resins even if the lignin fractions were insoluble in these solvents prior to the modification. The curing of each resin was verified by using FT-IR where the signals corresponding to thiol and ene had simultaneously been reduced after curing. One example is shown in Figure 4, and FT-IR spectra of all systems are shown in Figure S3.

Mechanical Properties. The mechanical properties of a polymeric thermoset material are determined by several factors that are derived from the chemical structure such as the mobility of the polymer backbone, the degree of crystallinity, intermolecular forces, the cross-linking density, dangling chain ends, and primary cycles (closed loops). In the case of lignin, the intrinsic heterogeneity of the molecular backbone does not allow crystalline structures to form, promoting a completely amorphous system. Dangling chain ends could be a result of an 


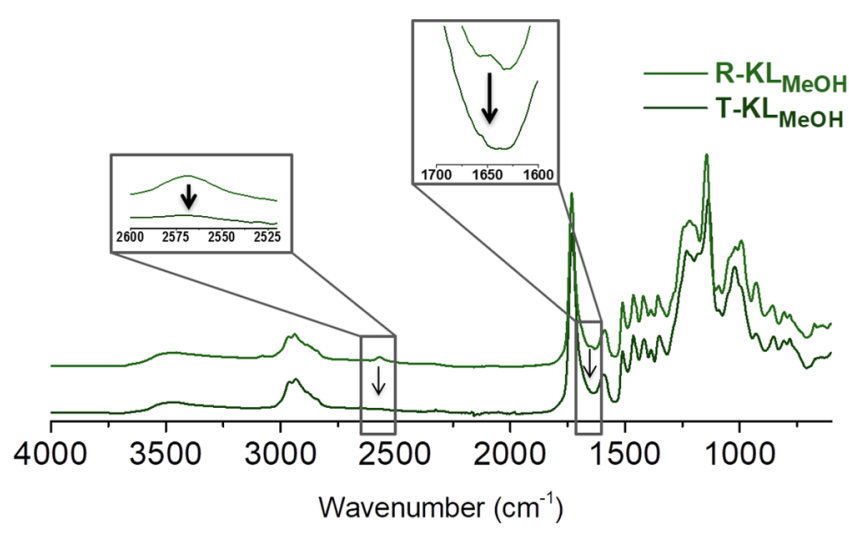

Figure 4. FT-IR spectra of the $\mathrm{KL}_{\mathrm{MeOH}}$-based resin $\left(\mathrm{R}-\mathrm{KL}_{\mathrm{MeOH}}\right)$ and thermoset $\left(\mathrm{T}-\mathrm{KL}_{\mathrm{MeOH}}\right)$. The signals corresponding to the thiol and ene $\left(2568\right.$ and $1647 \mathrm{~cm}^{-1}$ ) were heavily reduced as the resin underwent curing.

imperfect network where the cross-linking reaction did not reach full conversion. These dangling ends could act as plasticizers to a certain degree, lowering the glass transition temperature, $T_{\mathrm{g}}$, of the final material.

The cured thermoset samples were tested by using DMA to investigate the mechanical properties of the material. All DMA curves for the different measurements of the thermoset materials are found in Figure S4. These tests were performed in triplicates or quadruplicates, and no significant differences between repetitions were found, suggesting a good reproducibility of the different thermoset formulations.

Aromatic components in conventional thermosets, such as phthalate or bisphenol units, are in most cases rather small and evenly distributed within a polymer network. The lignin used in this study, however, consists of larger phenolic segments with a size distribution. How these entities contribute to the thermoset properties is far from evident; however, the results from the mechanical analysis indicate that there is a significant effect on the material properties depending on the lignin fraction used. There are a few parameters that can influence the $T_{g}$, for example, the molecular weight of the aromatic segments, the cross-linking density, and the possibility for internal interactions by secondary forces. The cross-linking density of the material will restrict the molecular movement, thus increasing the $T_{\mathrm{g}}$. Another factor that can influence the $T_{\mathrm{g}}$ is the ability to interact through secondary forces within the network, e.g., hydrogen bonding or $\pi-\pi$ stacking. A larger molecule incorporating more aromatic units would have a larger possibility to interact with other lignin segments through such secondary forces, thus increasing the $T_{\mathrm{g}}$. The degree of hydroxyl functionalities remaining in the network will also play a part through internal hydrogen bonding. This agrees well with the data on aliphatic alcohols and carboxylic acids presented in Figure 2.

The different shape, amplitude, and width of the Tan $\delta$ signal between the different thermosets provided information regarding the material properties. The $\operatorname{Tan} \delta$ signals of the studied thermosets are represented in Figure 5. The Tan $\delta$ is defined by the loss modulus $\left(E^{\prime \prime}\right)$ divided by the storage modulus $\left(E^{\prime}\right)$ and its maximum represents the largest change in molecular mobility and can be defined as the $T_{\mathrm{g}}$ of the material. However, the softening point of the material, where the $E^{\prime}$ starts to decrease substantially, will be observed at a lower temperature and could be considered as an engineering $T_{\mathrm{g}}$. In this work, the $T_{\mathrm{g}}$ derived from $\operatorname{Tan} \delta$ is the one in focus when describing the differences between the thermoset samples. A narrower Tan $\delta$ signal with a larger amplitude suggests that a sample is more homogeneous and has fewer defects compared to a sample with a broader signal with a lower amplitude. It is clearly seen that the lignin fractions of lower molecular weight exhibiting a narrower $Ð$ (Figure 1) yield a narrower signal with larger amplitudes than the thermosets based on the fractions of higher molecular weight and a larger $Đ$. These shapes of the Tan $\delta$ signals of the different thermosets correlate well with the dispersity data obtained from the SEC measurements. Density measurements of the different thermosets show no significant differences between the thermosets, regardless of the fraction used as a base for it. They all end up at around $1.3 \mathrm{~g} / \mathrm{cm}^{3}$. The physical properties of the different thermosets are presented in Table 1.

The storage modulus of the different thermosets below $T_{\mathrm{g}}$ is in the GPa region for all samples where the values at $-50{ }^{\circ} \mathrm{C}$, $E^{\prime}-50^{\circ} \mathrm{C}$, are presented for a fair comparison between them. These values are comparable to thermoset resin systems used in e.g. composite applications and indicate that lignin-based thermoset have a potential as a renewable candidate for these kinds of high-performance materials. ${ }^{11}$

The temperature where $E_{\text {min }}^{\prime}$ is reached is high enough so that secondary forces, such as hydrogen bonding and $\pi-\pi$ interactions, are broken, and the molecular backbone chain experiences maximum flexibility. The material would flow if it
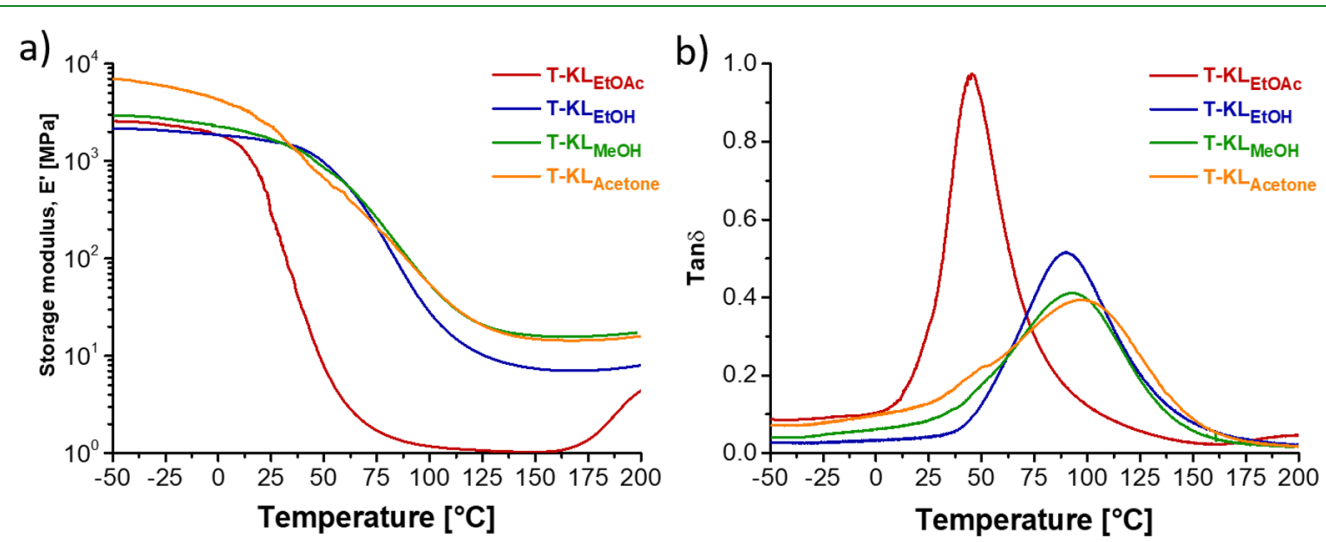

Figure 5. Storage modulus $E^{\prime}(\mathrm{a})$ and $\operatorname{Tan} \delta$ signals (b) of the different thermosets. The signal maximum is when the thermoset system experiences the highest loss rate and can be defined as the $T_{\mathrm{g}}$ of the material. 
Table 1. Physical Properties of the Thermosets: $T_{\mathrm{g} \text { Tan } \delta}$, Maximum and Minimum Storage Modulus, and Densities

\begin{tabular}{|c|c|c|c|c|}
\hline & $\mathrm{T}-\mathrm{KL}_{\mathrm{EtOAc}}$ & $\mathrm{T}-\mathrm{KL}_{\mathrm{EtOH}}$ & $\mathrm{T}-\mathrm{KL}_{\mathrm{MeOH}}$ & $\mathrm{T}-\mathrm{KL}_{\text {Acetone }}$ \\
\hline$T_{\mathrm{g} \operatorname{Tan} \delta}\left({ }^{\circ} \mathrm{C}\right)$ & $44 \pm 3$ & $95 \pm 3$ & $103 \pm 9$ & $94 \pm 3$ \\
\hline$E_{-50^{\circ} \mathrm{C}}^{\circ}[\mathrm{MPa}]$ & $2490 \pm 100$ & $1930 \pm 240$ & $3070 \pm 620$ & $5900 \pm 1990$ \\
\hline$E_{\min }^{\prime}[\mathrm{MPa}]$ & $1.4 \pm 0.7$ & $6.5 \pm 1.5$ & $19.6 \pm 7.1$ & $13.7 \pm 0.7$ \\
\hline$\rho_{\text {Thermoset }}\left[\mathrm{g} / \mathrm{cm}^{3}\right]$ & $1.28 \pm 0.01$ & $1.31 \pm 0.03$ & $1.31 \pm 0.03$ & $1.32 \pm 0.02$ \\
\hline
\end{tabular}

Table 2. Guinier Radius $R_{\mathrm{g}}$ As Determined from the SAXS Measurements and the $\pi-\pi$ Interaction within the Different Lignin Thermosets Where the Dimensions Are Extracted from the Observed WAXS Signals

\begin{tabular}{lllll} 
& $\mathrm{T}-\mathrm{KL}_{\mathrm{EtOAc}}$ & $\mathrm{T}-\mathrm{KL}_{\mathrm{EtOH}}$ & $\mathrm{T}-\mathrm{KL}_{\mathrm{MeOH}}$ & $\mathrm{T} \mathrm{KL}_{\mathrm{Acetone}}$ \\
$R_{\mathrm{g}}[\mathrm{nm}]$ & $4.4 \pm 0.1$ & $4.1 \pm 0.2$ & $3.8 \pm 0.2$ & $3.7 \pm 0.2$ \\
$\pi-\pi$ (sandwich) $[\AA]$ & $4.50 \pm 0.01$ & $4.58 \pm 0.01$ & $4.53 \pm 0.01$ & $4.62 \pm 0.01$ \\
& $84 \%$ & $95 \%$ & $90 \%$ & $94 \%$ \\
$\pi-\pi$ (T-shaped) $[\AA]$ & $7.23 \pm 0.1$ & $8.00 \pm 0.08$ & $7.52 \pm 0.06$ & $7.91 \pm 0.8$ \\
& $16 \%$ & $5 \%$ & $10 \%$ & $6 \%$ \\
\hline
\end{tabular}

Scheme 1. Representations of the Types of $\pi-\pi$ Interactions Present within the Thermosets As Suggested by the WAXS Measurement ${ }^{a}$
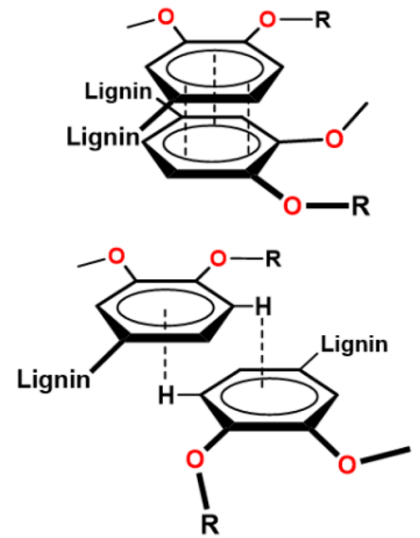

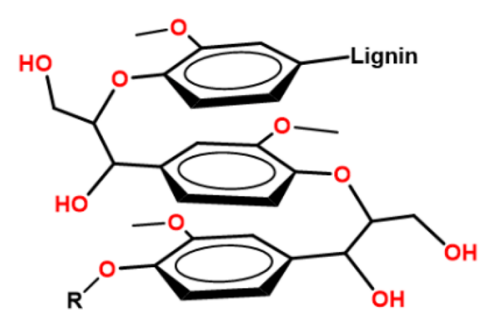

Intramolecular sandwich stacking in folded aryl ether structures

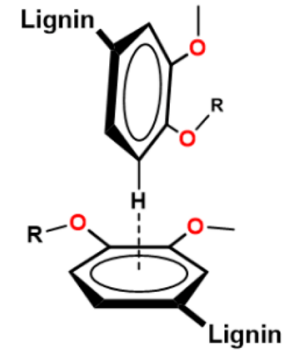

T-shape $\pi-\pi$ stacking

\section{Sandwich $\pi-\pi$ stackings}

${ }^{a}$ The intramolecular sandwich $\pi-\pi$ interactions by folding of aryl ether structures is also represented as suggested by Terashima et al. ${ }^{47}$ Some lateral displacements of the interactions can be expected.

was not hindered in a covalently bound network. $E_{\text {min }}^{\prime}$ occurs well above the $T_{\mathrm{g}}$ of the material and thus not at the same temperature for the different thermosets. From the DMA data it is clear that the structural properties of different fractions obtained from the LignoBoost Kraft lignin largely affect the final properties of the material. In the $\mathrm{A}-\mathrm{KL}_{\mathrm{EtOAc}}$ fraction the molecular weight is rather low and is likely to incorporate monofunctional molecules, with respect to allyl ethers, that will covalently bind to the network but not contribute to it as a whole and rather act as loose ends. When the fractions have higher molecular weight, the probability that some molecules would be monofunctional is drastically decreased. Thus, the leap in $T_{\mathrm{g}}$ between $\mathrm{T}-\mathrm{KL}_{\mathrm{EtOAc}}\left(M_{\mathrm{w}} \approx 1000 \mathrm{~g} / \mathrm{mol}\right)$ and $\mathrm{T}$ $\mathrm{KL}_{\mathrm{EtOH}}\left(M_{\mathrm{w}} \approx 2700 \mathrm{~g} / \mathrm{mol}\right)$ becomes significant, but there is no significant difference when using the fractions with even higher molecular weight. This would also explain why the $E_{\text {min }}^{\prime}$ is significantly lower for the $\mathrm{KL}_{\mathrm{EtOAc}}$-based thermoset compared to the others.

Lignin Thermoset Morphology. Producing thermosets from lignin oligomers and polymers will result in a molecular buildup different from traditional low molecular weight pure monomers. Within the network there will be heterogeneous lignin segments covalently bound to each other by the homogeneous cross-linker. This result in a two-phase system on an angstrom to nanometer level and is something completely different from traditional thermosets.

The Guinier radius, $R_{g}$, was extracted from the integrated one-dimensional scattering data for each thermoset and is shown in Table 2. The $R_{\mathrm{g}}$ describes domains of repeating features within the material, meaning that a smaller $R_{\mathrm{g}}$ represents a less homogeneous material. A larger $R_{g}$ indicated larger domains with repeating homogeneous size and similar polymer compositions extracted from the electron densities. The SAXS patterns and the one-dimensional plots of all thermosets are presented in Figures S5 and S6. The size of the $R_{\mathrm{g}}$ is slightly decreasing from around $4.4 \pm 0.1$ to $3.7 \pm 0.2 \mathrm{~nm}$ from $\mathrm{T}-\mathrm{KL}_{\mathrm{EtOAc}}$ to $\mathrm{T}-\mathrm{KL}_{\text {Acetone }}$ thermosets, i.e., with increasing $M_{\mathrm{w}}$, respectively. The dispersity, $Ð$, obtained from the SEC measurement (Figure 1), the Tan $\delta$ results (Table 1), and the extracted $R_{\mathrm{g}}$ values strongly suggest that the $\mathrm{T}-\mathrm{KL}_{\mathrm{EtOAc}}$ is the most homogeneous material consisting of larger repeating features. The thermosets incorporating the larger $M_{\mathrm{w}}$ lignin fractions (larger than $\mathrm{KL}_{\mathrm{EtOH}}$ ) seems to exhibit sizes that are more constant, approaching a value of about $3.7 \pm 0.2 \mathrm{~nm}$.

The WAXS patterns could all be fitted using two distinct Gaussian functions suggesting two reoccurring size domains in 
the region; the distributions are shown in Table 2. These corresponds well to typical $\pi-\pi$ stacking distances in sandwich and the perpendicular $\mathrm{T}$-shaped formations previously theoretically shown by Sinnokrot et al. for benzene dimers. ${ }^{44}$

Similarly, distributions like these have been shown by using WAXS for polymer blends including Kraft lignin ${ }^{45}$ and lignosulfonates. ${ }^{46}$ The dimensions of $\pi-\pi$ sandwich stacking are also in agreement with what has been reported for intramolecular sandwich stacking in folded native lignin hexalignols in aryl ether structures, where a distance of $4 \AA$ is reported as the most compact sandwich stacking in these structures. ${ }^{47}$ The distributions of the stackings are similar when using the higher molecular weight samples; however, the lowest molecular weight lignin yields a material with a higher relative number of $\mathrm{T}$-shaped stackings. A recent study of the structure of the same lignin fractions by Olsén et al. shows that there is a higher abundance of aryl ethers in the higher $M_{\mathrm{w}}$ fractions. ${ }^{48}$ This may in part explain the higher prevalence of sandwich stacking in the thermosets based on the higher $M_{\mathrm{w}}$ lignin. The aromatic rings of the lignin structures are likely to be found in these favorable $\pi-\pi$ stacking configurations, represented in Scheme 1, within each thermoset material. The WAXS patterns and Gaussian fits of the one-dimensional plots are found in Figures S7 and S8.

Thermal Degradation. TGA measurements and the thermal stability of the different samples reveal that the thermosets starts to degrade between 250 and $280{ }^{\circ} \mathrm{C}$. After full pyrolysis, when only char and ash remain, there are between 50 and 35 wt \% of residues remaining. More detailed information and the degradation curves of the thermosets are found in the Supporting Information (Figure S9).

\section{CONCLUSIONS}

All different LignoBoost Kraft lignin fractions could be selectively modified by using the allylation procedure previously developed and be cured by thermally induced thiol-ene cross-linking to produce freestanding thermoset samples for material analysis.

The properties, in terms of $T_{\mathrm{g}}, E^{\prime}$, and $E^{\prime \prime}$, of the final thermosets differ depending on the fraction used. The largest shift in $T_{\mathrm{g}}$ occurs between the thermoset based on the lowest molecular weight $\left(\mathrm{T}-\mathrm{KL}_{\mathrm{EtOAc}}\right)$ and the three others. This is likely due to the fact that the lowest molecular weight fraction includes smaller molecular segments with a monofunctional nature, which in turn do not contribute to the thermoset network but act more like dangling chain ends. The resulting lower cross-linking density is also the reason why the storage moduli becomes very low for this thermoset.

The lower the molecular weight and the smaller $Ð$, the more homogeneous materials with fewer defects are formed as seen in the $\operatorname{Tan} \delta$ curves in the DMA measurements. The results achieved through SAXS measurements strengthen this claim through small differences in $R_{\mathrm{g}}$. These results suggest that the $\mathrm{T}-\mathrm{KL}_{\mathrm{EtOAc}}$ is the most homogeneous thermoset produced in this study.

The WAXS measurements indicate different ratios of $\pi-\pi$ stacking. T-KL $L_{\mathrm{EtOAc}}$ shows a higher relative amount of $\mathrm{T}$ shaped $\pi-\pi$ stacking compared to the thermosets based on larger molecular weight lignin.

The TGA measurements shows that the materials are stable up to $250{ }^{\circ} \mathrm{C}$ under a nitrogen atmosphere and that the ash contend after thermal degradation is very different between the different thermosets.
These findings are a crucial step toward understanding how these kinds of material network are built up and how fractionation of lignin affects both material properties and morphologies. The study provides insight into what each fraction brings to these material systems and allows for further evaluation of the process depending on the final application toward reproducible and predictable material properties. It is necessary to understand these aspects if one would like to utilize lignin as a raw material in sustainable thermoset applications. One such application could be as matrices in composite materials where these results show suitable properties.

\section{ASSOCIATED CONTENT}

\section{SI Supporting Information}

The Supporting Information is available free of charge at https://pubs.acs.org/doi/10.1021/acsapm.9b01007.

The fractionation procedure and related SEC results, details and descriptions of the ${ }^{31} \mathrm{P}$ NMR procedure, ${ }^{1} \mathrm{H}$ and ${ }^{31} \mathrm{P}$ NMR spectra of the lignin fractions as well as the selectively allylated products and the calculated degree of allylation, FT-IR spectra of the resins as well as the cured thermosets of each sample and their compositions, DMA curves of all samples as well as the SAXS and WAXS scattering patterns and the $1 \mathrm{~d}$ plots with corresponding fitting and TGA discussion points and results (PDF)

\section{AUTHOR INFORMATION}

\section{Corresponding Author}

Mats Johansson - Wallenberg Wood Science Center and Division of Coating Technology, Department of Fibre and Polymer Technology, KTH Royal Institute of Technology, 100 44 Stockholm, Sweden; 이이이.org/0000-0003-3201-5138; Email: matskg@kth.se

\section{Authors}

Marcus E. Jawerth - Wallenberg Wood Science Center and Division of Coating Technology, Department of Fibre and Polymer Technology, KTH Royal Institute of Technology, 100 44 Stockholm, Sweden

Calvin J. Brett - Wallenberg Wood Science Center, Division of Coating Technology, Department of Fibre and Polymer Technology, and Department of Engineering Mechanics, KTH Royal Institute of Technology, 10044 Stockholm, Sweden; Deutsches-Elektronen Synchrotron (DESY), 22607 Hamburg, Germany; 이이이.org/0000-0001-5789-6299

Cédric Terrier - Division of Coating Technology, Department of Fibre and Polymer Technology, KTH Royal Institute of Technology, 10044 Stockholm, Sweden; Department of Materials Science and Engineering, SMX, EPFL - Ecole Polytechnique Féderale de Lausanne, 1015 Lausanne, Switzerland

Per T. Larsson - Wallenberg Wood Science Center, KTH Royal Institute of Technology, 10044 Stockholm, Sweden; RISE Bioeconomy, 11486 Stockholm, Sweden

Martin Lawoko - Wallenberg Wood Science Center, KTH Royal Institute of Technology, 10044 Stockholm, Sweden; (1) orcid.org/0000-0002-8614-6291

Stephan V. Roth - Division of Coating Technology, Department of Fibre and Polymer Technology, KTH Royal Institute of Technology, 10044 Stockholm, Sweden; Deutsches-Elektronen 
Synchrotron (DESY), 22607 Hamburg, Germany;

(1) orcid.org/0000-0002-6940-6012

Stefan Lundmark - Perstorp AB, Innovation, 28480 Perstorp, Sweden

Complete contact information is available at:

https://pubs.acs.org/10.1021/acsapm.9b01007

\section{Notes}

The authors declare no competing financial interest.

\section{ACKNOWLEDGMENTS}

The authors are grateful to the Knut and Alice Wallenberg Foundation for financial support through the Wallenberg Wood Science Center at KTH Royal Institute of Technology. The authors acknowledge beam time allocation at PETRA III, DESY (Hamburg, Germany). C.J.B. and S.V.R. acknowledge the kind financial support from the DESY strategic fund (DSF) "Investigation of processes for spraying and spray-coating of hybrid cellulose-based nanostructures". DESY is a member of the Helmholtz Association (HFG). We thank Samer Nameer for helpful discussions regarding the DMA machine and Carmen Cobo Sánchez with the suggestion to do, and the help to perform, TGA measurements.

\section{ABBREVIATIONS}

$\mathrm{KL}$, LignoBoost Kraft lignin; $\mathrm{KL}_{\text {Fractiont }}$ general fraction soluble in a certain solvent (fraction = EtOAc, $\mathrm{EtOH}, \mathrm{MeOH}$, or acetone); A- $\mathrm{KL}_{\text {Fraction, }}$ the allylated product of a specific lignin fraction; $\mathrm{R}-\mathrm{KL}_{\text {Fraction }}$ the $\mathrm{A}-\mathrm{KL}_{\text {Fraction }}$ mixed with cross-linker; $\mathrm{T}-\mathrm{KL}_{\text {Fraction, }}$ the formed thermoset after curing of $\mathrm{R}-\mathrm{KL}_{\text {Fraction}}$.

\section{REFERENCES}

(1) Stewart, D. Lignin as a base material for materials applications: Chemistry, application and economics. Ind. Crops Prod. 2008, 27, 202.

(2) Duval, A.; Lawoko, M. A review on lignin-based polymeric, micro- and nano-structured materials. React. Funct. Polym. 2014, 85, 78.

(3) Lora, J. H.; Glasser, W. G. Recent Industrial Applications of Lignin: A Sustainable Alternative to Nonrenewable Materials. J. Polym. Environ. 2002, 10, 39.

(4) Wang, M.; Wang, F. Catalytic Scissoring of Lignin into Aryl Monomers. Adv. Mater. 2019, 31, 1901866.

(5) Tran, C. D.; Chen, J.; Keum, J. K.; Naskar, A. K. A New Class of Renewable Thermoplastics with Extraordinary Performance from Nanostructured Lignin-Elastomers. Adv. Funct. Mater. 2016, 26, 2677.

(6) Lallave, M.; Bedia, J.; Ruiz-Rosas, R.; Rodríguez-Mirasol, J.; Cordero, T.; Otero, J. C.; Marquez, M.; Barrero, A.; Loscertales, I. G. Filled and Hollow Carbon Nanofibers by Coaxial Electrospinning of Alcell Lignin without Binder Polymers. Adv. Mater. 2007, 19, 4292.

(7) Föllmer, M.; Jestin, S.; Neri, W.; Vo, V. S.; Derré, A.; Mercader, C.; Poulin, P. Wet-Spinning and Carbonization of Lignin-Polyvinyl Alcohol Precursor Fibers. Adv. Sustainable Syst. 2019, 3, 1900082.

(8) Tenhaeff, W. E.; Rios, O.; More, K.; McGuire, M. A. Highly Robust Lithium Ion Battery Anodes from Lignin: An Abundant, Renewable, and Low-Cost Material. Adv. Funct. Mater. 2014, 24, 86.

(9) Van Krevelen, D. W. In Properties of Polymers, 3rd completely revised ed.; Elsevier: Amsterdam, 1997; Chapter 1, p 3.

(10) Van Krevelen, D. W. In Properties of Polymers, 3rd, completely revised ed.; Elsevier: Amsterdam,1997; Chapter 6, p 129.

(11) Åström, B. T. Manufacturing of Polymer Composites, new ed.; Nelson Thornes: Cheltenham, 2002.

(12) Boerjan, W.; Ralph, J.; Baucher, M. Lignin Biosynthesis. Annu. Rev. Plant Biol. 2003, 54, 519.
(13) Chakar, F. S.; Ragauskas, A. J. Review of current and future softwood kraft lignin process chemistry. Ind. Crops Prod. 2004, 20, 131.

(14) Crestini, C.; Lange, H.; Sette, M.; Argyropoulos, D. S. On the structure of softwood kraft lignin. Green Chem. 2017, 19, 4104.

(15) Hu, Z.; Du, X.; Liu, J.; Chang, H.; Jameel, H. Structural Characteriztion of Pine Kraft Lignin: Biochoice Lignin vs Indulin AT. J. Wood Chem. Technol. 2016, 36, 432.

(16) Lancefield, C. S.; Wienk, H. L. J.; Boelens, R.; Weckhuysen, B. M.; Bruijnincx, P. C. A. Identification of a diagnostic structural motif reveals a new reaction intermiediate and condensation pathway in kraft lignin formation. Chem. Sci. 2018, 9, 6348.

(17) Tomani, P. The LignoBoost Process. Cellul. Chem. Technol. 2010, 44, 53.

(18) Duval, A.; Vilaplana, F.; Crestini, C.; Lawoko, M. Solvent screening for the fractionation of industrial kraft lignin. Holzforschung 2016, 70, 11.

(19) Jääskeläinen, A. S.; Liitiä, T.; Mikkelson, A.; Tamminen, T. Aqueous organic solvent fractionation as means to improve lignin homogeneity and purity. Ind. Crops Prod. 2017, 103, 51.

(20) Wallberg, O.; Jönsson, A. S.; Wimmerstedt, R. Fractionation and concentration of kraft black liquor lignin with ultrafiltration. Desalination 2003, 154, 187.

(21) Toledano, A.; García, A.; Mondragon, I.; Labidi, J. Lignin separation and fractionation by ultrafiltration. Sep. Purif. Technol. 2010, 71, 38

(22) Prat, D.; Wells, A.; Hayler, J.; Sneddon, H.; McElroy, C. R.; Abou-Shehada, S.; Dunn, P. J. CHEM21 selection guide of classicaland less classical-solvents. Green Chem. 2016, 18, 288.

(23) Baumberger, S.; Lapierre, C.; Monties, B.; Valle, G. D. Use of kraft lignin as filler for starch films. Polym. Degrad. Stab. 1998, 59, 273.

(24) Gordobil, O.; Delucis, R.; Egüés, I.; Labidi, J. Kraft lignin as filler in PLA to improve ductility and thermal properties. Ind. Crops Prod. 2015, 72, 46.

(25) Ikeda, Y.; Phakkeeree, T.; Junkong, P.; Yokohama, H.; Phinyocheep, P.; Kitano, R.; Kato, A. Reinforcing biofiller "Lignin" for high performance green natural rubber nanocomposites. RSC Adv. 2017, 7, 5222.

(26) Wang, C.; Kelley, S. S.; Venditti, R. A. Lignin-Based Thermoplastic Materials. ChemSusChem 2016, 9, 770.

(27) Jawerth, M.; Johansson, M.; Lundmark, S.; Gioia, C.; Lawoko, M. Renewable Thiol-Ene Thermosets Based on Refined and Selectively Allylated Industrial Lignin. ACS Sustainable Chem. Eng. 2017, 5, 10918.

(28) Gioia, C.; Lo Re, G.; Lawoko, M.; Berglund, L. Tunable Thermosetting Epoxies Based on Fractionated and Well-Characterized Lignins. J. Am. Chem. Soc. 2018, 140, 4054.

(29) Buono, P.; Duval, A.; Averous, L.; Habibi, Y. Lignin-Based Materials Through Thiol-Maleimide "Click" Polymerization. ChemSusChem 2017, 10, 984.

(30) Over, L. C.; Hergert, M.; Meier, M. A. R. Metathesis Curing of Allylated Lignin and Different Plant Oils for the Preparation of Thermosetting Polymer Films with Tunable Mechanical Properties. Macromol. Chem. Phys. 2017, 218, 1700177.

(31) Jawerth, M.; Lawoko, M.; Lundmark, S.; Perez-Berumen, C.; Johansson, M. Allylation of a lignin model phenol: a highly selective reaction under benign conditions towards a new thermoset resin platform. RSC Adv. 2016, 6, 96281.

(32) Odian, G. Principles of Polymerization; Wiley: 2004

(33) Dušek, K. Are cured thermoset resins inhomogeneous? Angew. Makromol. Chem. 1996, 240, 1.

(34) Kloosterboer, J. G. Network formation by chain crosslinking photopolymerization and its applications in electronics. In Advances in Polymer Science. Springer: Berlin, 1988.

(35) Hoyle, C. E.; Lee, T. Y.; Roper, T. Thiol-enes: Chemistry of the past with promise for the future. J. Polym. Sci., Part A: Polym. Chem. 2004, 42, 5301.

(36) Hoyle, C. E.; Bowman, C. N. Thiol-Ene Click Chemistry. Angew. Chem., Int. Ed. 2010, 49, 1540. 
(37) Chang, B.; Schneider, K.; Xiang, F.; Vogel, R.; Roth, S. V.; Heinrich, G. Critical Strains for Lamellae Deformation and Cavitation during Uniaxial Stretching of Annealed Isotactic Polypropylene. Macromolecules 2018, 51, 6276.

(38) Yu, S.; Chen, F.; Wu, Q.; Roth, S. V.; Brüning, K.; Schneider, K.; Kuktaite, R.; Hedenqvist, M. S. Structural Changes of Gluten/ Glycerol Plastics under Dry and Moist Conditions and during Tensile Tests. ACS Sustainable Chem. Eng. 2016, 4, 3388.

(39) Argyropoulos, D. S. Quantitative Phosphorus-31 NMR Analysis of Lignins, a New Tool for the Lignin Chemist. J. Wood Chem. Technol. 1994, 14, 45.

(40) Buffet, A.; Rothkirch, A.; Dohrmann, R.; Korstgens, V.; Abul Kashem, M. M.; Perlich, J.; Herzog, G.; Schwartzkopf, M.; Gehrke, R.; Muller-Buschbaum, P.; Roth, S. V. P03, the microfocus and nanofocus $\mathrm{X}$-ray scattering (MiNaXS) beamline of the PETRA III storage ring: the microfocus endstation. J. Synchrotron Radiat. 2012, 19, 647.

(41) Benecke, G.; Wagermaier, W.; Li, C.; Schwartzkopf, M.; Flucke, G.; Hoerth, R.; Zizak, I.; Burghammer, M.; Metwalli, E.; MullerBuschbaum, P.; Trebbin, M.; Förster, S.; Paris, O.; Roth, S. V.; Fratzl, P. A customizable software for fast reduction and analysis of large Xray scattering data sets: applications of the new DPDAK package to small-angle X-ray scattering and grazing-incidence small-angle X-ray scattering. J. Appl. Crystallogr. 2014, 47, 1797.

(42) Jiang, Z.; Tang, Y.; Rieger, J.; Enderle, H. F.; Lilge, D.; Roth, S. V.; Gehrke, R.; Wu, Z.; Li, Z.; Li, X.; Men, Y. Structural evolution of melt-drawn transparent high-density polyethylene during heating and annealing: Synchrotron small-angle X-ray scattering study. Eur. Polym. J. 2010, 46, 1866.

(43) Balakshin, M.; Capanema, E. On the Quantification of Lignin Hydroxyl Groups With 31P and 13C NMR Spectroscopy. J. Wood Chem. Technol. 2015, 35, 220.

(44) Sinnokrot, M. O.; Valeev, E. F.; Sherrill, C. D. Estimates of the $\mathrm{Ab}$ Initio Limit for $\pi-\pi$ Interactions: The Benzene Dimer. J. Am. Chem. Soc. 2002, 124, 10887.

(45) Li, Y.; Sarkanen, S. Miscible Blends of Kraft Lignin Derivatives with Low-Tg Polymers. Macromolecules 2005, 38, 2296.

(46) Wang, Y. Y.; Chen, Y. R.; Sarkanen, S. Path to plastics composed of ligninsulphonates (lignosulfonates). Green Chem. 2015, $17,5069$.

(47) Terashima, N.; Yoshida, M.; Hafrén, J.; Fukushima, K.; Westermark, U. Proposed supramolecular structure of lignin in softwood tracheid compound middle lamella regions. Holzforschung 2012, 66, 907.

(48) Olsén, P.; Jawerth, M.; Lawoko, M.; Johansson, M.; Berglund, L. A. Transforming technical lignins to structurally defined starcopolymers under ambient conditions. Green Chem. 2019, 21, 2478. 